\title{
Philosophiques
}

\section{Les études wittgensteiniennes au Canada : état de la recherche, 1970-1984}

\section{François Latraverse}

Volume 12, numéro 1, printemps 1985

URI : https://id.erudit.org/iderudit/203280ar

DOI : https://doi.org/10.7202/203280ar

Aller au sommaire du numéro

Éditeur(s)

Société de philosophie du Québec

ISSN

0316-2923 (imprimé)

1492-1391 (numérique)

Découvrir la revue

Citer cet article

Latraverse, F. (1985). Les études wittgensteiniennes au Canada : état de la recherche, 1970-1984. Philosophiques, 12(1), 197-209.

https://doi.org/10.7202/203280ar d'utilisation que vous pouvez consulter en ligne.

https://apropos.erudit.org/fr/usagers/politique-dutilisation/ 
PhILOSOPhIQues, Vol. XII, Numéro 1, Printemps 1985

\title{
BULLETIN
}

\section{LES ÉTUDES WITTGENSTEINIENNES AU CANADA : ÉTAT DE LA RECHERCHE, 1970-1984}

\author{
par François Latraverse
}

La rubrique "Bulletin " de Philosophiques a été occupée, de manière assez irrégulière, depuis les origines de la revue, par des études portant sur des activités de recherche organisés autour de la pensée d'un philosophe. La philosophie de Wittgenstein en est venue à s'imposer au responsable de la rubrique comme ayant assez d'importance pour mériter d'être traitée.

La fortune de la pensée de Wittgenstein demeure assez étrange : ayant longtemps, dans certains milieux, été identifiée a priori comme un objet de méfiance radicale, elle est souvent devenue le lieu d'une exploitation forcenée, qui s'autorise de la richesse de cette pensée pour y trouver surtout ce qu'elle y apporte. Dans d'autres, elle a été limitée à ces traits qui la rendaient compatibles avec un certain nombre d'idées établies, alors qu'elle comporte plusieurs aspects qui s'accommodent mal aux paradigmes habituels. Dans d'autres milieux enfin, elle demeure l'objet d'une curiosité lointaine, comme si, tout en reconnaissant son importance sur le terrain de l'histoire de la philosophie et sur celui des problématiques actuelles, on se contentait d'enregistrer intuitivement sa richesse et sa pertinence sans prendre la peine de voir ce qu'il en est. Il est vrai que la parution et plus encore la diffusion assez tardives d'œuvres autres que le Tractatus logico-philosophicus et les Recherches philosophiques ont laissé à un commentaire simplificateur le temps de s'instaurer et de figer en un certain nombre de lieux communs ce qu'on semblait pouvoir attendre de cette philosophie.

Au Québec, c'est, me semble-t-il, autour de 1965 que l'existence de la pensée de Wittgenstein a commencé d'être enregistrée dans les institutions francophones. Wittgenstein y est alors apparu comme un des chefs de file d'un mouvement encore mal identifié, mais jugé différent des façons habituelles de pratiquer la philosophie, savoir le mouvement dit de la philosophie analytique. Pour diverses raisons, ce mouvement a connu une implantation relativement lente et controversée. Je ne peux approfondir ici les difficultés de cette résistance, qui peut maintenant, selon les points de vue, apparaître saugrenue et lointaine ou fondée et proche, mais il vaut la peine d'insister sur le fait que placer Wittgenstein comme porte-étendard d'un mouvement aussi nuancé et diversifié que la philosophie analytique aurait dû aller et devrait continuer d'aller moins de soi que ce qui s'est souvent trouvé être le cas. L'idée devenue banale qui consiste à dire que Wittgenstein a successivement fondé et répudié deux des écoles les plus prospères de la philosophie 
contemporaine (pour le dire rapidement, le positivisme logique et la philosophie dite du langage ordinaire) mériterait, dans ses diverses implantations territoriales, d'être examinée pour elle-même. La position qu'occupe Wittgenstein quant à ces écoles gagnerait à être précisée à cet égard.

Les données qui suivent ont cherché à composer une image aussi représentative que possible de l'état des études wittgensteiniennes au Canada au cours des quatorze dernières années. Ces données sont groupées selon les catégories suivantes : publications (livres et articles consacrés en tout ou en partie à Wittgenstein), conférences et communications, mémoires et thèses, recherches et enseignement, catégories qui sont apparues constituer les principaux paramètres d'identification de l'importance que la pensée de Wittgenstein trouve maintenant dans les institutions canadiennes. Le collectage et l'organisation de ces données ont rencontré les difficultés inhérentes à ce genre d'entreprises.

Une difficulté pratique générale a trait au rassemblement des données : le dépouillement des revues, des programmes des colloques et congrès, des répertoires de cours, etc. a dû être relayé par une enquête faite auprès des départements de philosophie du Québec et de l'ensemble du Canada. Le caractère plus ou moins complet de la compilation est fonction directe des réponses reçues, surtout pour ce qui est des activités des wittgensteinisants canadiens à l'étranger.

Par ailleurs, les catégories retenues n'imposent pas des critères ayant tous la même netteté : s'il est raisonnablément simple de reconnaître un article ou un livre portant directement et nommément sur la philosophie de Wittgenstein, l'identification de textes portant partiellement sur elle est plus délicate. Sur bon nombre des questions majeures qui se posent à la philosophie contemporaine, les positions de Wittgenstein sont de plus en plus difficiles à éviter, de sorte que le recensement des mentions qui en sont faites ou des travaux influencés par elles risquerait de rassembler une part de la production philosophique bien supérieure à ce qui est souhaitable et pratiquement compilable. Cette difficulté frappe également la nomenclature des conférences et communications et plus encore celui des mémoires et thèses. La pensée de Wittgenstein ayant été une influence exercée sur la recherche au moins autant qu'un objet de recherche, le relevé de ses marques a dû faire la part des utilisations implicites et des utilisations explicites. Au nombre de tous les éléments susceptibles d'être retenus à un titre ou à un autre, j'ai cherché à retenir (en particulier dans les rubriques des livres et des articles partiellement consacrés à Wittgenstein) ceux dans lesquels cette pensée joue un rôle de premier plan, plutôt que de n'y fournir qu'un mode de traitement parmi d'autres. Malgré cet effort, le découpage comporte forcément une part d'arbitraire.

Dans le cas des activités d'enseignement, les informations données demeurent très partielles. S'il semble que la plupart des programmes de premier, de deuxième et de troisième cycles au Canada fassent une place à la pensée de Wittgenstein, il est souvent difficile d'en établir l'ampleur. Il 
n'est pas rare, aussi bien dans les institutions anglophones que dans les institutions francophones, que les descriptions des cours et séminaires qui sont présentées dans les programmes soient très ouvertes et qu'il soit confié aux versions offertes d'un trimestre à l'autre d'en définir les contenus particuliers. Lorsque des informations précises étaient disponibles, elles ont été intégrées, mais on ne peut conclure de leur absence pour une institution donnée à l'inexistence d'enseignements portant sur Wittgenstein dans cette institution. Sur ce plan aussi bien que sur les autres, les efforts du compilateur peuvent être déjoués par ce qu'il faut bien appeler l'inertie des milieux concernés. Ceci est une façon (bien indirecte, je l'admets) de remercier les personnes qui ont bien voulu prêter leur concours à la courte étude que je présente.

Département de philosophie

Université du Québec à Montréal

Articles :

BLACKWELL, Kenneth, "The Early Wittgenstein and the Middle Russell", in BLOCK, I., éd., Perspectives on the Philosophy of Wittgenstein, pp. 130 .

BLOCK, Irving, "Showing in the Tractatus : the Root of Wittgenstein and Russel's Basic Incompatibility", Russell, 17, 1975, pp. 4-22.

"Hart on the Whole-Sense of the Tractatus", International Logic Review, 12, 1975, pp. 145-57.

“The Unity of Wittgenstein's Philosophy", 4th International Wittgenstein Symposium, Vienne, Hölder-Pichler-Tempsky, 1979, pp. 233-36.

c.r. de Vesey, G., Understanding Wittgenstein, Philosophia, 7, 1980, pp. 717-33.

BURNS, S.A.M., cr. de Pears, David, Wittgenstein, Dialogue, 11, 1972, pp. 478-80.

c.r. de Wittgenstein, Ludwig, Vermischte Bemerkungen, Dialogue, 21, 1982, pp. 178-81.

CANFIELD, John V., "A Model Tractatus Language", The Philosophical Forum, 4, 1972-73, pp. 199-217.

"Criteria and Method", Metaphilosopby, 5, 1974, pp. 298-315.

"Criteria and Rules of Language", Philosophical Review, 83, 1974, pp. 70-89.

"Wittgenstein and Zen", Philosophy, 50, 1975, pp. 383-408.

"Anthropological Science Fiction and Logical Necessity", Canadian Journal of Philosophy, 4, 1975, pp. 467-79.

"Tractatus Objects", Philosophia, 6, 1976, pp. 81-99. 
“ 'I know that I am in Pain' is Senseless", in Lehrer, K., éd., Analysis and Metaphysics, Dordrecht, 1977, pp. 129-44.

c.r. de Diamond, Cora, éd., Wittgenstein's Lectures on the Foundations of Mathematics, Canadian Journal of Philosophy, 2, 1981.

CENTORE, FF., "A Note on Wittgenstein as an Unwilling Nominalist", The Thomist, 37, 1973, pp. 762-67.

ENGLEBRETSEN, Georg, "Behaviorism and Perception", Man and World, 7, 1974, pp. 149-57.

"What in the World is the Truth about Logical Space ?" Philosophical Inquiry, IV, 1982, pp. 52-61.

"Do We Need Relative Identity ?", Notre Dame Journal of Formal Logic, 23, 1982, pp. 91-93.

GRENNAN, Wayne, "Wittgenstein on Religious Utterances" Sopbia, 15, 1976, pp. 13-18.

GRIFFIN, Nick, "Wittgenstein, Universals, and Family Ressemblances", Canadian Journal of Pbilosophy, 3, 1974, pp. 635-51.

HUNTER, J.F.M., "Wittgenstein and Knowing the Meaning of a Word", Dialogue, 10, 1971 , pp. 294-304.

"Wittgenstein on Describing and Making Connexions, The Philosophical Quarterly, 26, 1976, pp. 243-50.

"Why Animals Don't Talk", Dialogue, 15, 1976, pp. 290-95.

"Some Grammatical States", Philosophy, 1977, pp. 155-66.

"Wittgenstein on Inner Processes and Outward Criteria", Canadian Journal of Philosophy, 7, 1977, pp. 805-17.

"Wittgenstein and Materialism", Mind, 86, 1977, pp. 514-31.

“A Scholars's Wittgenstein”, Philosophical Review, 87, pp. 259-74.

"Wittgenstein on Language and Games", Philosophy, 55, 1980, pp. 293-302.

"Wittgenstein on Seeing and Seeing As", Philosopbical Investigations, 4, 1981, pp. 33-49.

"Wittgenstein's Remarks on the Philosopby of Psychology", Canadian Philosophical Review, 1, 1981, pp. 130-42.

c.r. de Hacker, P.M.S., Insight and Illusion, Canadian Journal of Philosopby, 1974, pp. 201-11.

c.r. de Wittgenstein, Ludwig, The Philosophy of Psychology, Queen's Quarterly, 1982, pp. 842-49.

c.r. de Wittgenstein's Lectures, Cambridge 1930-32 et de Wittgenstein's Lectures, Cambridge 1932-35, Canadian Journal of Philosophy, 1984, pp. 153-65.

JOHNSON, Ralph H., "Wittgenstein : Philosophy and Grammar", Proceeaings of the American Catbolic Pbilosophical Association, 44, 1970, pp. 99107. 
LAPOINTE, Roger, «L'écart des deux Wittgenstein comme discours ", Dialogue, 10, 1971, pp. 276-93.

LATRAVERSE, François, «Ce que se taire veut dire : Remarques sur la question du silence dans le Tractatus ", Presses Universitaires de France, Corps écrit, $\mathrm{N}^{\circ} 12,1984$, pp. 39-54. Version préliminaire dans Archives et Documents, Société d'Histoire et d'Épistémologie des Sciences du Langage, Paris, 1984.

"Les gardiens du sens : Remarques sur Wittgenstein et le symbolique ", Montréal, HMH, Sédiments (à paraître).

"Sprachspiele und Rationalität ", in Verabschiedung der (Post-)Moderne? Eine kontroverse Dokumentation, WUNBERG, G. et al., éd., Tübingen, Gunter Narr Verlag, (à paraître, 1985).

"Rituels sans objets : Remarques sur Wittgenstein et l'anthropologie ", L'efficacité du symbolique, UQAM, (à paraître).

«La nécessité de l'arbitraire. Remarques sur la question de l'analyticité chez Wittgenstein ", Nadeau, Robert, éd., Contingence et raison. Essais sur la controverse de l'analyticité, Québec, Presses de l'Université du Québec (à paraître).

NIELSEN, Kai, "The Coherence of Wittgenstein's Fideism", Sopbia, 11, pp. 4-12.

"The Challenge of Wittgenstein. An Examination of his Picture of Religious Belief', Studies in Religion, 3, pp. 29-46.

"Remarks on Wittgensteinian Method. An Examination of J.F.M. Hunter's Essays after Wittgenstein, Metapbilosophy, 7, pp. 241-64.

POULAIN, Jacques, «Le mysticisme du Tractatus Logico-Pbilosophicus et la situation paradoxale des propositions religieuses ", La recherche en philosophie et en théologie, Paris, Éd. du Cerf, 1970, pp. 75-155.

"La possibilité des propositions ontologiques dans le Tractatus logicophilosophicus ", Logique et philosophie, Les Études philosophiques, 4, 1973, pp. 529-52.

ROSE, Robert, c.r. de Winch, Peter, éd., Studies in the Pbilosophy of Wittgenstein, Dialogue, 9, 1970, pp. 268-273.

SCHACHTER, J.P., "The Private Language Passages", Canadian Journal of Pbilosophy, 12, 1982, pp. 479-94.

SHINER, Roger A., "Wittgenstein's Philosophy of Language", Dialogue, 12, 1973, pp. 683-99.

"Wittgenstein on the Beautiful, the Good and the Tremendous", British Journal of Aesthetics, 14, 1974, pp. 258-71.

"Wittgenstein and the Foundations of Knowledge", Proceedings of the Aristotelian Society, 78, 1977-78, pp. 103-24.

"Canfield, Cavell and Criteria", Dialogue, 22, 1983, pp. 253-72.

SZABADOS, Béla, “Wittgenstein on Belief', Philosopbical Papers, 1981, pp. 24-34. 


\footnotetext{
“Wittgenstein on Mistrusting One's Own Belief", Canadian Journal of Philosophy, 12, 1982, pp. 603-12.

c.r. de Mc Guinness, Brian, éd., Wittgenstein and His Times, Canadian Philosophical Review, 1984.
}

THIRY, L., c.r. de Novielli, Valeria, Wittgenstein e la filosofia, Dialogue, 10, 1971, pp. 207-09.

ZWICKY, Jan L., "Wittgenstein and the Logic of Inference", Dialogue, 21, 1982, pp. 671-92.

c.r. de Canfield, John V., Wittgenstein : Language and World, Canadian Journal of Philosophy (à paraître).

c.r. de Block, Irving, éd., Perspectives on the Pbilosopby of Wittgenstein, Dialogue, 23, 1984, pp. 357-61.

Articles partiellement consacrés à Wittgenstein :

BONNET, Jacques et F. LATRAVERSE, «Un message impérial », L'Arc, 67, 1976, pp. 64-80.

CROMBIE, James E., "Peirce on Our Knowledge of Mind : A Neglected Third Approach", in Caws, Peter, éd., Two Centuries of Pbilosopby in America, Oxford, Basil Blackwell, 1980, pp. 77-85.

FRENCH, Stanley, "Kant's Constitutive-Regulative Distinction", in Kant Studies Today, Beck, L.W., éd., Lasalle, Open Court, 1970, pp. 37591.

« Droit à la sécession ", Philosophie et Droit, Montréal et Paris, Bellarmin et Desclée, 1979, pp. 85-99.

FRENCH, Stanley et A. GUTMAN, "The Principle of National Selfdetermination", in Philosophy, Morality and International Affairs, Nagel, T. et all, éds, Londres, Oxford University Press, 1974, pp. 138-53.

GAUTHIER, Yvon, c.r. de Bouveresse, Jacques, La parole malbeureuse, Dialogue, 11, 1972, pp. 480-81.

HUNTER, J.F.M., "Asking Oneself”, Philosophical Investigations, 1/3, 1978, Pp. 14-24.

"Believing", Midwest Studies in Philosophy, 5, 1980, pp. 239-60.

“Talking to Oneself', Dialogue, 21, 1982, pp. 111-23.

KAUFMANN, J.N., " Philosophie analytique de l'action et fondement normatif des sciences de l'homme, Dialogue, 23, 1984, pp. 3-35.

LATRAVERSE, F. et Suzanne LEBLANC, "On the Delimitation of Semantics and the Characterization of Meaning : Some Remarks", Possibilities and Limitations of Pragmatics, PARRET, H., M. SBISÀ et J. VERSCHUEREN, éds., Amsterdam, John Benjamins B.V., Studies in Language Companion Series, 7, 1981, pp. 399-412.

McCORMICK, P., SCHAPER, E., HEATON, S.M., "Symposium on Saying and Showing in Heidegger and Wittgenstein", Journal British Soc. Phenomenol., 3, 1972, pp. 27-45. 
NIELSEN, Kai, "Sociological Knowledge : Winch, Marxism, and Versteben Revisited", Philosophy and Phenom. Research, pp. 465-91.

"Wisdom and Dilman on the Scope of Reason in Religion", Philosophical Investigations, 3/4, 1980, pp. 1-14.

"On the Rationality of Groundless Believing", Idealistic Studies, 11, 1981, pp. 215-29.

ODEGARD, D., "Two Types of Scepticism”, Philosophy, 54, 1979, pp. 46174.

POULAIN, Jacques, «Problèmes logiques du discours philosophique », Les Quatre fleuves, 6, 1976, pp. 49-64.

"La critique de la religion dans la philosophie analytique " et « Jeux de langage et actes de discours religieux ", Tendances principales de la recherche dans les sciences sociales et bumaines, tome II, La Haye-Paris, Mouton-Unesco, 1979 , pp. $1532-37$ et $1540-42$.

Monographies, ouvrages collectifs :

BLOCK, Irving, éd., Perspectives on the Philosopby of Wittgenstein, Oxford, Basil Blackwell, 1981, 322 p.

CANFIELD, John V., Wittgenstein : Language and World, Amherst, The University of Massachusetts Press, 1981, $230 \mathrm{p}$.

HUNTER, J.F.M., Essays after Wittgenstein, Toronto, University of Toronto Press, 1973, 202 p.

Understanding Wittgenstein, Edimbourg, Edinburgh University Press, (à paraître, 1985), $\pm 300 \mathrm{p}$.

How To Look on Words as Instruments, 1984, 80000 mots, 1984, (en quête d'éditeur).

KAAL, Hans et McKINNON, Alastair, Concordance to Wittgenstein's Philosophische Untersuchungen, Leiden, E.J.Brill, 1975, xiii et 596 p.

POULAIN, Jacques, Logique et religion. L'atomisme logique de Wittgenstein et la possibilité des propositions religieuses, suivi de Logic and Religion : a shortened and adapted version, La Haye-Paris, Mouton, 1973, 228 p.

Monographies partiellement consacrées à Wittgenstein :

CRADDOCK, C.A., Mondrian, Pound, Wittgenstein : Affinities, Montréal, Mansfield Book Mart Ltd., 1978, 11 p.

HACKING, Ian, Why Does Language Matter to Pbilosophy ?, Cambridge, Cambridge University Press, 1975, en part. chap. 8.

HUNTER, J.F.M., Intending, a Monograph Series, Halifax, 1978, 74 p.

LOPTSON, P.J., "Introductory Essay" à The Priciples of the Most Ancient and Modern Philosophy de Anne Conway, La Haye, Martinus Nijhoff, 1982.

ODEGARD, D., Knowledge and Scepticism, Rowman \& Littlefield, 1982. 
Mémoires et thèses :

ASHLEY, Wayne T., Wittgenstein's Critique of Moore's Epistemology, Université de Windsor, 1983.

BELL, David A., Wittgenstein's Theory of the Self, Universite McMaster, 1971.

BIENERT, Ronald F., "That Which is Shown" as the Unifying Project in Wittgenstein's Philosophy, Université McMaster, 1984.

BILCAR, Branko, Wittgenstein on the Grammar of Knowledge-Claims, Université de Windsor, 1982.

BITTLE, Kevin W., Dreaming : An Investigation into the Grammar of Traditional and Contemporary Problems Associated with Dreaming, Université de Windsor, 1982.

CLARET, Anne-Marie, $D u$ jeu de langage au jeu du langage : Wittgenstein, Derrida et la crise des fondements en philosopbie, Université du Québec à Montréal, mémoire en cours.

DELVAUX, Marie-Claire, Sens et non-sens chez Wittgenstein et Heidegger, Université de Montréal, 1973.

FOISY, Suzanne, Sens, absolu et logique chez Wittgenstein, Université de Montréal, 1973.

FROHMANN, Bernard, Wittgenstein on Proper Names, Université de Toronto, 1981.

GARDNER, Susan, A Person's Concept of Person : A Developmental Model of Self, Université Concordia, 1981.

GRANT, Grell V., The Relevance of Psychoanalysis to Pbilosophy : The View of Morris Lazerowitz, Université Concordia, 1978.

The Linguistic Turn in Psychoanalysis : The View of Roy Schafer, Université Concordia, 1984.

GRAVES, Peter R., The Private Language Argument, Université McMaster, 1974.

HENDERSON, Connie, Wittgenstein, Kant and Transcendental Pbilosophy, Université Concordia, 1974.

HILMY, Stephen, Philosopbical Metbod in the Later Work of Wittgenstein, Université de Toronto, 1985.

HUTCHINSON, William B., Popper and Rationality : A Wittgensteinian Critique, Université de Windsor, 1981.

KELLY, Robert J., The Consistency of Wittgenstein's Conception of the Nature of Philosophy, Université Concordia, 1974.

KOGGEL, C., Paradigms : the Later Wittgenstein's View of Meaning, Université Carleton, 1981.

KOWALSKY, Borys M., Wittgenstein and Philosophy, Université McMaster, 1982. 
LABERGE, Jean, La notion de " grammaire " chez Wittgenstein, Université du Québec à Montréal, 1983.

La philosophie des mathématiques de Ludwig Wittgenstein, Université du Québec à Trois-Rivières, thèse en cours.

LEMAY, Diane, Du rapport entre les ,ints et les choses : essai de philosophie du langage, Université de Sherbrooke, 1976.

LESSARD, Adèle, Les jeux de langage freudiens, Université de Montréal, 1975.

MACKENSIE, Michael, Showing and Saying in the Philosophical Investigations, Université Concordia, 1978.

MIGHTON, John, Imagination as Method : Section xii, Part II of Wittgenstein's Philosophical Investigations, Université McMaster, 1982.

MURPHY, Norman, Le problème de la signification chez Wittgenstein, Université du Québec à Trois-Rivières, 1977.

OFFENBACH, E., Wittgenstein's Conception of Meaning, Université McGill, 1975.

PLAMONDON, Jacques, La conception du langage chez Wittgenstein, Université d'Aix-Marseille, 1970.

POULAIN, Jacques, Pragmatique et ontologie, Université de Nantes, 1984.

RAPHAEL, L., Wittgenstein et son rapport à Hertz, Université McGill, 1978.

ROCHESTER, Judith, Pbilosopby as Therapy : An Examination of Wittgenstein's Philosophical Method, Université de Toronto, 1978.

RUSSEL, W. Benjamin, Wittgenstein's Grammar, Université de Toronto, 1982.

SOUCY, Gaëthan, Articulation des usages de la raison dans la machine critique : structure et signification de la philosophie transcendantale de Kant, [appendice sur l'aprioricité de l'espace et la forme du monde chez Kant et Wittgenstein], Université du Québec à Montréal, 1985.

STALL, Rosanna, Wittgenstein's Willing Subject, Université Concordia, 1975.

STEVENS, Laird, Meaning, Université Concordia, 1982.

TEAYS, Wanda, Naming the Unnamable : Language and Silence in Beckett's Novels, Université Concordia, 1980.

TREMBLAY, Richard, Sens et non-sens chez Wittgenstein, Université de Montréal, 1971.

VANDERVEKEN, Daniel, Le problème de la signification dans le Tractatus Logico-philosophicus, Université de Louvain, 1970.

\section{Conférences et communications :}

BILODEAU, Renée, "Le rôle des énoncés à propos des états psychologiques dans la justification de l'action ", 28 Congrès de l'Association Canadienne de Philosophie, Guelph, juin 1984. 
BLOCK, Irving, "Russell and Wittgenstein", Colloque Russell, Université McMaster, novembre 1973.

"Russell, Pears and Others on the Tractatus - A History of Misinterpretation", Université Vanderbilt, février 1974.

Russel and Wittgenstein", $18^{e}$ Congrès de l'Association Canadienne de Philosophie, Université de Toronto, mai 1974.

"Wittgenstein and the Sceptic", $27^{\mathrm{e}}$ Congrès de l'Association Canadienne de Philosophie, Montréal, août 1983 et Université Princeton, 1984.

BROOK, J.A., "Two Second Paralogisms", $21^{\mathrm{e}}$ Congrès de l'Association Canadienne de Philosophie, Fredericton, juin 1977.

BURNS, S., "If The Lion Could Talk . . .", $28^{\mathrm{e}}$ Congrès de l'Association Canadienne de Philosophie, Guelph, juin 1984. Comm. par B. Szabados.

CANFIELD, John, MULLET, Sheila, et RESNICK, D., "Symposium on Wittgenstein : Language and World de J. Canfield, 26 Congrès de l'Association Canadienne de Philosophie, Ottawa, juin 1982.

FRENCH, Stanley, "Wittgenstein", Centre de recherche en histoire des idées, Université de Nice, 1976.

GRENNAN, Wayne, "Winch, Wittgenstein and the Justification of Language Games", $23^{e}$ Congrès de l'Association Canadienne de Philosophie, Saskatoon, juin 1979.

HOTTOIS, Gilbert, KAUFMANN, Nicolas, et LATRAVERSE, François, « Nouvelles perspectives dans la philosophie de Wittgenstein ", table ronde, $17^{\mathrm{e}}$ Congrès Mondial de Philosophie, Montréal, août 1983.

HUNTER, J.F.M., “A Fork in the Road”, University of Oregon, mars 1977. "Wittgenstein on Depth Grammar", S.U.N.Y. Buffalo at Amherst, mai 1977.

"What Wittgenstein Means by 'Depth Grammar' ", 26e Congrès de l'Association Canadienne de Philosophie, Ottawa, juin 1982. Comm. par M. Faghfoury.

KAVANAGH, R., Representation: Foucault and Wittgenstein", $15^{\mathrm{e}}$ Congrès de l'Association Canadienne de Philosophie, St.Jean, T.N., juin 1971.

KAUFMANN, J. Nicolas, "Control and Intentional Causation in Analytical Philosophy of Action", 7th International Wittgenstein Symposium, Kirchberg a. W., août 1984.

LABERGE, Jean, «Le principe de négation signifiante " $27^{\mathrm{e}}$ Congrès de l'Association Canadienne de Philosophie, Montréal, août 1983. Comm. par F. Latraverse.

"L'idée de preuve chez Wittgenstein : Remarques sur le " finitisme " de Wittgenstein ", 28 ${ }^{\mathrm{e}}$ Congrès de l'Association Canadienne de Philosophie, Guelph, juin 1984. Comm. par J. Couture. 
LATRAVERSE, François, «Grammaire et actes de langage », Congrès de l'ACFAS, Montréal, mai 1979.

« Grammaire et philosophie », Université du Québec à Montréal, février 1981.

"Pragmatique et iconicité », Congrès de l'Association Canadienne de Sémiotique, Montréal, juin 1981.

" Jeux de langage et critique de la raison », Maison des Sciences de l'Homme, Paris, mai 1983.

«La conceptualisation de la pragmatique ", Université de Grenoble, mai 1983.

«Ce que se taire veut dire : Remarques sur Tractatus 7 ", $27^{\mathrm{e}}$ Congrès de l'Association Canadienne de Philosophie, Montréal, août 1983. Comm. par N. Kaufmann. Version remaniée présentée au colloque «Le silence ", Université de Nice, juin 1984.

"Rituels sans objets : Wittgenstein et l'anthropologie ", Colloque "L'efficacité du symbolique ", Université du Québec à Montréal, avril 1984.

"Vienne, Wittgenstein et la modernité », Société de philosophie de Montréal, février 1984.

«La conventionnalité de la convention ", $3^{\text {e }}$ Congrès de l'Association Internationale de Sémiotique, Palerme, juin 1984.

LEMAY, Diane, "Mind-body Problem : The Corporal and Social Elaboration of the Mental", 7th Annual Ludwig Wittgenstein Symposium, Kirchberg a. W., août 1984.

LUGG, Andrew, "Science and the Primacy of Practice", Workshop on Methodological Rules, Virginia Polytechnic Institute, avril 1984.

McKINNON, Alastair, "Kierkegaard and Wittgenstein", Université de Gronningen, novembre 1982.

"A Problem in Wittgenstein's On Certainty", $17^{\mathrm{e}}$ Congrès Mondial de Philosophie, Montréal, août 1983.

MULLET, Sheila et John CANFIELD, "Wittgenstein Workshop", $20^{\mathrm{e}}$ Congrès de l'Association Canadienne de Philosophie, Québec, juin 1976.

NIELSEN, H.A., "Realism, Nominalism and Wittgenstein", $23^{\mathrm{e}}$ Congrès de l'Association Canadienne de Philosophie, Saskatoon, juin 1979. Comm. par B. Szabados.

NIELSEN, Kai, "John Hunter after Wittgenstein", $18^{\mathrm{e}}$ Congrès de l'Association Canadienne de Philosophie, U. de Toronto, juin 1974. Comm. par J.F.M. Hunter.

"La Longue Marche à travers les institutions : de Wittgenstein à Marx ", Allocution présidentielle, $28^{\mathrm{e}}$ Congrès de l'Association Canadienne de Philosophie, Guelph, juin 1984.

RAPHAEL, L., "Points de contact entre Wittgenstein et Kierkegaard ", $16^{\mathrm{e}}$ Congrès de l'Association Canadienne de Philosophie, Université McGill, juin 1972. 
RESNICK, L., "Some Doubts about Skepticism", 5th Annual Ludwig Wittgenstein Symposium, Kirchberg a. W., août 1982.

"Gappy Causation", 6th Annual Ludwig Wittgenstein Symposium, Kirchberg a. W., août 1983.

"Justification and Personal Identity", 7th Annual Ludwig Wittgenstein Symposium, Kirchberg a. W., août 1984.

SHINER, R.A., "Wittgenstein on the Good and the Beautiful", $18^{\mathrm{e}}$ Congrès de l'Association Canadienne de Philosophie, U. de Toronto, juin 1974. Comm. par J.D.B. Walker.

SZABADOS, Béla, "Wittgenstein on mistrusting one's own belief', $24^{\mathrm{e}}$ Congrès de l'Association Canadienne de Philosophie, Université du Québec à Montréal, juin 1980. Comm. par L. Resnick.

\section{Recherches :}

McKINNON, Alastair et Hans KAAL, Creation of Index Verborum and Concordance to Published Works of Wittgenstein", CRSH (1977), CRSH (1980), McGill (1983), CRSH (1983).

KAUFMANN, Nicolas, François LATRAVERSE, Renée BILODEAU et Jean LABERGE, "Contribution de la philosophie analytique de l'action aux recherches fondationnelles en sciences de l'homme ", FCAC (198385).

LATRAVERSE, François et Walter MOSER, «Vienne au tournant du siècle » (À propos de la culture viennoise 1890-1930), projet Littérature comparée (Université de Montréal) et Philosophie (Université du Québec à Montréal).

ZWICKY, J.L., "Wittgenstein's Metaphysics", recherche post-doctorale, CRSHC, 1983-84.

\section{Enseignement :}

Université Carleton : un cours de $3^{\mathrm{e}}$ année sur le Tractatus et les Recherches philosophiques. Un cours de niveau gradué sur De la certitude et les Fiches.

Université McMaster : deux cours de niveau gradué : Philosophy 735 (Wittgenstein : The Early Writings) et 736 (Wittgenstein : The Later Writings).

Université de Montréal : si aucun cours ne porte explicitement sur Wittgenstein, bonne place lui est faite dans de nombreux cours, dont : " Philosophie du langage ", "Pragmatique du langage » et "Philosophie analytique ».

Université d'Ottawa : en dépit du caractère discret avoué de la pensée de Wittgenstein dans les programmes, un cours consacré aux Recherches philosophiques est parfois offert et un cours régulier consacré à la philosophie angloaméricaine contemporaine fait une place considérable à Wittgenstein. 
Université du Québec à Trois-Rivières : aucun cours n'est nommément consacré à Wittgenstein, mais celui-ci occupe une place importante dans les cours " Positivisme » et "Philosophie des sciences de l'homme » au $2^{\mathrm{e}}$ cycle, "La philosophie analytique ", "L'empirisme logique », " La philosophie au $\mathrm{XX}^{\mathrm{e}}$ siècle » au $1^{\mathrm{er}}$ cycle. Un cours « Textes d'auteurs en philosophie contemporaine " a été entièrement consacré au Tractatus en 1978 et un séminaire de doctorat a eu lieu en 1983 sur «Wittgenstein, philosophie de la psychologie et thèmes voisins".

Université du Québec à Montréal : un cours de premier cycle (PHI 4019) est entièrement consacré à Wittgenstein. Plusieurs enseignements de $1^{\mathrm{er}}, 2^{\mathrm{e}}$ et $3^{\mathrm{e}}$ cycles lui font en outre une place importante, en particulier : " Philosophie analytique ", "Méta-éthique ", "Philosophie de l'action ", "Pragmatique ".

Université de Saskatchewan : un séminaire sous-gradué sur la philosophie de Wittgenstein dans son ensemble.

Université de Waterloo : un séminaire gradué est consacré à Wittgenstein.

Université de Windsor : les cours de niveau sous-gradué 34-436 (Contemporary British Philosophy), 34-211 (Philosophy of Language) et 34-326 (Philosophy of Religious Language) font une part importante à Wittgenstein. Au niveau gradué, le cours 34-517 lui est explicitement consacré. 